\title{
Bioimpedância em doenças infecciosas: revisão crítica
}

\section{Bioimpedance in infectious diseases: critical review}

\author{
Naise Oliveira da Rocha ${ }^{1}\left[\right.$ (D) , Abelardo de Queiroz Campos Araújo ${ }^{1}$ (D), Paula Simplício da Silva ${ }^{1}$ (D) Paloma Ribeiro Torres ${ }^{1}$ (D) ,

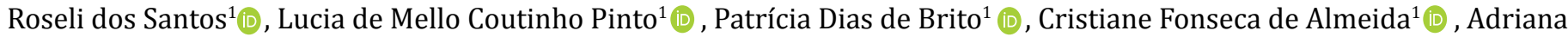 \\ Costa Bacelo ${ }^{1}$
}

1. Instituto Nacional de Infectologia de Evandro Chagas - (INI/FIOCRUZ), Rio de Janeiro, RJ, Brasil.

\begin{abstract}
Resumo
Objetivo: verificar se o ângulo de fase, obtido por bioimpedância elétrica, pode ser utilizado como indicador prognóstico em doenças infecciosas e em quais dessas doenças seu uso está adequadamente embasado pela literatura científica. Métodos: revisão integrativa realizada por meio das bases de dados, como google acadêmico, na BVS Brasil, nas bases SciELO, LILACS e Pubmed, utilizando o termo para busca (bioimpedância e doenças infectocontagiosas e bioimpedância) AND (bioimpedance and infectious diseases OR bioimpedance). A seleção dos estudos foi feita, considerando artigos originais completos disponíveis on-line, em inglês, espanhol e português, publicados entre 2007 e 2021. Resultados: todos os estudos considerados (793) foram realizados em adultos com doença infecciosa. Destes $28(3,5 \%)$ foram separados para leitura aprofundada sobre o perfil metodológico, e apenas quatro (0,50\%) do total de artigos consideraram o ângulo de fase como índice prognóstico para doenças infeciosas, ambos em pacientes HIV + hospitalizados. Conclusão: A bioimpedância vem sendo considerada como instrumento de avaliação de estado nutricional em pacientes com doenças infecciosas. Mas o uso do ângulo de fase vem sendo pouco estudado como índice prognóstico para essa população, não podendo ser considerado adequadamente embasado para uso clínico na população com doença infecciosa, o que suscita maior atenção a esta população e a necessidade de maior investigação científica.
\end{abstract}

Palavras-chave: Doenças Infecciosas. Estado Nutricional.

\begin{abstract}
Objective: to verify if the phase angle obtained by electrical bioimpedance can be used as a prognostic indicator in infectious diseases and in which infectious diseases its use is adequately supported by scientific literature. Methods: integrative review conducted using databases such as Google Scholar, BVS Brazil, SciELO, LILACS and Pubmed, using the search term (bioimpedance and infectious diseases and bioimpedance) AND (bioimpedance and infectious diseases OR bioimpedance). The selection of studies was made considering complete original articles available online, in English, Spanish and Portuguese, published between 2007 and 2021. Results: all studies considered (793) were carried out in adults with infectious disease. Of these, 28 (3.5\%) were separated for in-depth reading on the methodological profile, and only four $(0.50 \%)$ of the total articles considered the phase angle as a prognostic index for infectious diseases, both in hospitalized HIV + patients. Conclusion: Bioimpedance has been considered as an instrument to assess nutritional status in patients with infectious diseases. However, the use of the phase angle has been little studied as a prognostic index for this population, and cannot be considered adequately substantiated for clinical use in the population with infectious disease, which raises more attention to this population and the need for further scientific investigation.
\end{abstract}

Keywords: Infectious Diseases. Nutritional Status.

\section{INTRODUÇÃO}

O estado nutricional e a imunidade estão intimamente ligados, facilitando ou dificultando a ocorrência de doenças infeciosas ${ }^{1}$.

As doenças infecciosas podem causar febre, fadiga, diarreia, mialgia, emagrecimento, astenia, alterações fisiológicas, bioquímicas e histopatológicas ${ }^{2,3}$. A relação entre a má nutrição e as doenças infecciosas, em países em desenvolvimento, é conhecida, sendo a má nutrição capaz de agravar o quadro clínico e aumentar as chances de morte ${ }^{1,4,5}$.

A Bioimpedância (BIA) é uma ferramenta de diagnóstico nutricional já estabelecida na prática clínica, principalmente em pacientes acamados, fornecendo o ângulo de fase (AF), que vem sendo considerado como indicador prognóstico em pacientes críticos, por se relacionar com o equilíbrio celular e ter grande afinidade como indicador de gravidade de doença ${ }^{6-9}$.

Dessa forma, o presente estudo pretende verificar se o AF pode ser utilizado como indicador prognóstico em doenças infecciosas, assim como revisar em quais doenças infecciosas seu uso já está adequadamente embasado pela literatura científica.

\section{METODOS}

Foi realizada uma revisão crítica integrativa de estudos envolvendo o uso da bioimpedância na avaliação do estado nutricional e o ângulo de fase como indicador prognóstico. A pesquisa foi realizada em março de 2021 e inclui artigos publicados entre junho de 2007 e abril de 2021, nas seguintes 
bases de dados: Google Acadêmico, Biblioteca Virtual em Saúde (BVS Brasil), nas bases Scientific Eletronic Library On-line (SciELO), Literatura Latino-Americana e do Caribe em Ciências da Saúde (LILACS) e Medical Literature Analysis and Retrieval System Online (Medline Pubmed).

Para a estratégia de busca, foram utilizados os seguintes descritores: "bioimpedância", "doenças infectocontagiosas", "HTLV", "bioimpedance", "infectious diseases", os quais foram combinados por meio dos operadores booleanos OR e AND. A mesma estratégia de busca, trocando somente os aspectos relativos a cada doença avaliada, foi utilizada para HIV, HTLV, tuberculose, dengue e doença de Chagas. Foram investigados, para a revisão integrativa, todos os artigos publicados em português, inglês ou espanhol nos últimos 14 anos (junho de 2007 a abril de 2021).

Os resumos identificados foram analisados para selecionar estudos originais de base científica em humanos, cujos resultados exploravam o uso da bioimpedância como um indicador prognóstico no estado nutricional de pacientes com doenças infecciosas em fase aguda. Estudos experimentais e de revisão bibliográfica foram excluídos. E os artigos selecionados foram lidos na íntegra com a finalidade de selecionar os estudos adequados para compor esta revisão.

Os artigos lidos na íntegra foram selecionados, conforme a relevância do desenho e sua adequação ao tema. Nesse processo, foram excluídos os que não apresentavam, mesmo que, secundariamente, resultados específicos sobre a relação entre o uso da bioimpedância e sua capacidade prognóstica do estado nutricional em doenças infecciosas. Estudos eminentemente descritivos, baseados apenas em populações de doentes ou infectados (estudos de série de casos), também foram excluídos.

\section{RESULTADOS}

Foram encontrados 793 artigos nas bases de dados escolhidas para o estudo, seguindo as estratégias de busca descritas. Destes, 317 (39,97\%) foram excluídos após a filtragem por período do ano de 2007 a 2021. Os títulos e resumos dos demais $476(60,03 \%)$ artigos foram lidos, sendo retirados os que não tinham relação com doenças infecciosas, que realizavam a BIA em animais e os que citavam a BIA, mas não a utilizavam como referência ou como um indicador prognóstico; desse modo, foram excluídos 449 (56,62\%) artigos, e separados para leitura completa, 28 (3,53\%) artigos, excluindo, em seguida, mais 19 $(2,39 \%)$ artigos, por se aterem à definição de bioimpedância, sem discutir seu uso na população estudada.

Assim, somente 9 (1,26\%) artigos foram utilizados neste estudo.
Figura 1. Fluxograma da busca bibliográfica integrativa

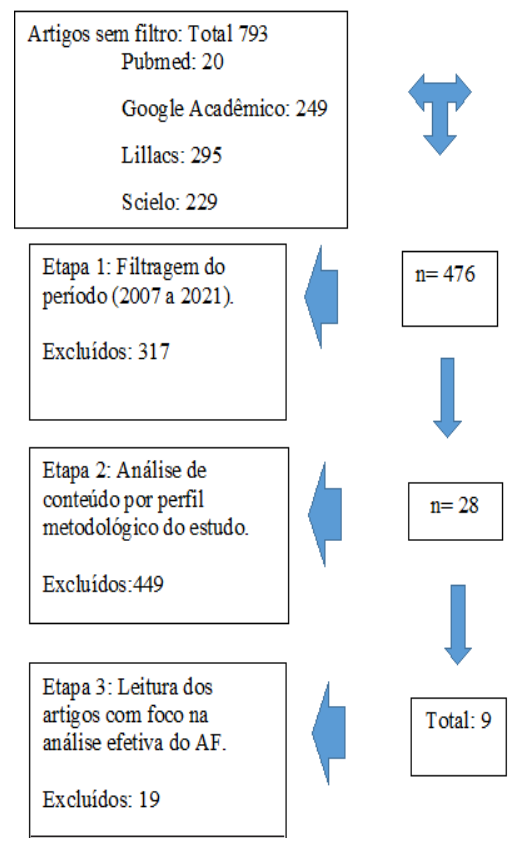

Destes nove estudos, cinco: Santos, D.K.S. $(2012)^{10}$, Matute P. P, et al. (2013) ${ }^{11}$, Santos DSK et al (2012) (10), Beraldo et.al $(2017)^{13}$, e Montalvo et al. $(2018)^{14}$, realizaram suas pesquisas em pessoas que vivem com HIV e utilizaram a BIA apenas para avaliação da composição corporal, não discutindo o valor de AF. Outro, Antunes (2012) ${ }^{15}$, também realizado com pessoas que vivem com HIV, não avaliou o estado nutricional, mas analisou os valores de $A F$, encontrando um de $A F$ de 5,30 em 51,4\% da população, o que sugere um pior prognóstico para essa população.

Apenas, León, D.J. A. et al $(2016)^{16}$, que também estudaram pessoas com HIV, utilizou a BIA para avaliação da composição corporal e verificação do AF como indicador prognóstico, sugerindo valores entre 6.15으 a 5.6ㅇ.

O único estudo encontrado com doenças infectocontagiosas que não foi associado ao HIV foi o realizado por Khalil; Mohktar, Ibrahim (2016) (17), que comparou o AF de indivíduos infectados com o vírus da dengue com e sem a manifestação da doença. $E$, apesar de não informar o valor do AF encontrado, aponta que os infectados adoecidos apresentaram um AF menor que o outro grupo comparado.

O estudo mais recente encontrado foi de SILVA; LIBONATI, $(2020)^{18}$ com paciente infectados com HIV / Aids apresentando uma média de 6,45으 de AF. Portanto, sugerindo que um AF menor está associado a alterações metabólicas múltiplas em HIV, e propondo seu uso como ferramenta de acompanhamento do estado nutricional e lipodistrofia. 
3 Bioimpedância em doenças infecciosas: revisão crítica

Quadro 1. Detalhamento dos artigos utilizados na revisão

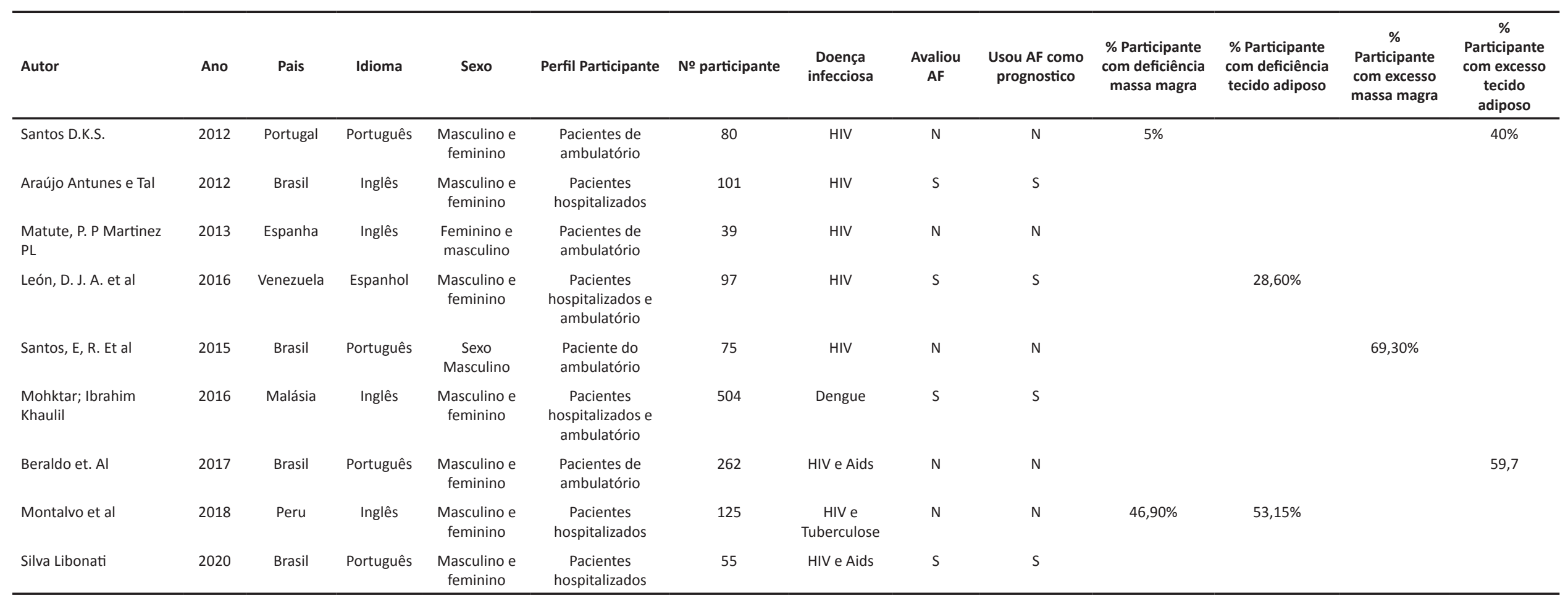




\section{DISCUSSÃO}

Os dados revisados neste documento sugerem que 1) a população com HIV vem sendo mais bem estudada quanto à composição corporal por bioimpedância, que as demais populações acometidas por doença infecciosa; 2) ainda são poucos os estudos com bioimpedância em doenças infecciosas; 3) não há estudos suficientes para embasar, adequadamente, um ponto de corte do uso do ângulo de fase por bioimpedância em pessoas com doenças infecciosas.

São poucos os estudos de composição corporal em população com doenças infecciosas, assertiva que é corroborada pela literatura $(19,20)$. Contudo, nosso estudo aponta para o fato de que a população com HIV vem sendo mais bem estudada quanto à composição corporal por BIA, que as demais populações acometidas por doença infecciosa; ou seja, em nossa busca de estudos de BIA e doenças infecciosas, não localizamos nenhum estudo de avaliação de composição corporal e AF por BIA em pacientes portadores de doenças de Chagas, infecção por HTLV, leishmaniose ou paracoccidioidomicose.

Os poucos estudos encontrados sugerem que pessoas que vivem com HIV tendem a apresentar maior percentual de tecido adiposo que a população em geral ${ }^{10,11,12,13,15,16,18}$ e que a BIA é um bom instrumento para tal investigação ${ }^{21}$.

Os dois únicos estudos com avaliação do AF sugerem que pessoas que vivem com HIV tendem a ter um AF menor que a população em geral, deixando a dúvida sobre a possibilidade de prejuízo na integridade celular ${ }^{7,22}$.

Vale ressaltar que os quatro artigos que utilizaram a BIA como indicador prognóstico estudaram pacientes hospitalizados desnutridos, sendo, portanto, esperado que o cálculo entre a reatância e resistência, que gera o AF, aponte para anormalidade e, por isso, era previsível que o AF se comportasse como um indicador de pior prognóstico para aquela população, limitando a discussão dos resultados 23,16,18. $^{2}$.

Mas o estudo com pessoas infectadas pelo vírus da dengue também descreve uma menor integridade de membrana, ou seja, menor $\mathrm{AF}$, em pacientes infectados sintomáticos, mostrando que a sintomatologia está associada com morte celular, valendo ressaltar que os autores não demostraram mediante a média de sua população e atração do $p$ valor sendo significativo ${ }^{17}$.

Dessa forma, esta revisão não identificou nenhum estudo que discuta, de forma contundente, a questão do valor prognóstico do $A F$, restringindo o uso da BIA em infectologia ao olhar da composição corporal. Portanto, verifica-se a necessidade de mais estudos relacionados com a utilização da BIA e da capacidade preditiva do AF em pacientes portadores de doenças infectocontagiosas.

\section{CONSIDERAÇÕES FINAIS}

A escassez de estudos sobre a bioimpedância e ângulo de fase em pacientes com doenças infecciosas ficou evidente nesta revisão, não sendo possível considerar o ângulo de fase como indicador prognóstico em doenças infeciosas; e, ainda, a literatura impõe a necessidade de mais estudos relacionados com a utilização da avaliação de bioimpedância em pacientes portadores de doenças infectocontagiosas, tanto com relação à composição corporal, quanto ao uso do ângulo de fase como indicador prognóstico.

\section{REFERÊNCIAS}

1. Paes NA. A mortalidade por doenças infecciosas e parasitárias na população idosa brasileira. Rev Panam Salud Publica. 2004; 15(4): :233-41.

2. Souza HP, Oliveira WTGH, Santos JPC, Toledo JP, Ferreira IPS, Esashika SNGS. Doenças infecciosas e parasitárias no Brasil de 2010 a 2017: aspectos para vigilância em saúde. Rev Panam Salud Publica. 2020; 44:e10. doi: 10.26633/ RPSP.2020.10

3. Reis FOB dos, Guimarães MSA, Chiacchio AD, Oliveira NA de. A doença em cada século: a influência do comportamento social nas principais pandemias dos últimos 200 anos. Rev Desafios. 2021; 8(1): 104-19. doi: http://dx.doi. org/10.20873/uftv8-9631.

4. Barros MB de A, César CLG, Carandina L, Torre GD. Desigualdades sociais na prevalência de doenças crônicas no Brasil, PNAD-2003. Ciênc saúde coletiva. 2006 Out-Dez; 11(4): 911-26. doi: http://dx.doi.org/10.1590/S141381232006000400014 .

5. Perrut JF, Santos EP, Oliveira BR, Carneiro ACLL, Oliveira GL, Ervilha AB Júnior, et al. Risco nutricional e sua associação com o tempo e desfecho da internação de pacientes com aids em um hospital de referência em infectologia de belo horizonte. Espaç. saúde. 2014 Abr;15(1): 57-65.

6. Eickemberg M, Sampaio LR, Oliveira CC de Anna Karla Carneiro R.
Bioimpedância elétrica e sua aplicação em avaliação nutricional. Rev nutr. 2011 Nov-Dez ;24(6):873-82. doi: https://doi.org/10.1590/S141552732011000600009 .

7. Llames L, Baldomero V, Iglesias ML, Rodota LP. Valores del ángulo de fase por bioimpedancia eléctrica: estado nutricional y valor pronóstico. Nutr Hosp. 2013 Mar-Abr; 28(2):286-95. doi: https://dx.doi.org/10.3305/nh.2013.28.2.6306.

8. Edwick D, Hince D, Rawlins J, Wood F, Edgar D. Bioimpedance Spectroscopy Is a Valid and Reliable Measure of Edema Following Hand Burn Injury (Part 1-Method Validation). J Burn Care Res. 2020 Jul; 41(4): 780-787. doi: 10.1093/ jbcr/iraa071.

9. Albert K, Delano M. This Whole Thing Smacks of Gender": Algorithmic Exclusion in Bioimpedance-based Body Composition Analysis. Digital library . 2021 Mar. 342-352. https://doi.org/10.1145/3442188.3445898.

10. Santos DSK Avaliação do Estado Nutricional em doentes com VIH/SIDA. 2012. 1-20 f. Dissertação (Trabalho acadêmico). [Portugual]: Universidade do Porto; 2012.doi: https://repositorio-aberto.up.pt/handle/10216/68895.

11. Pérez-Matute P, Pérez-Martínez L, Blanco JR, Ibarra V, Metola L, Sanz M, et al. Multiple frequency bioimpedance is an adequate tool to assess total and regional fat mass in HIV-positive patients but not to diagnose HIV-associated 
lipoatrophy: a pilot study. J Int AIDS Soc. 2013 Dec; 16(1): 18609. doi: 10.7448/ IAS.16.1.18609.

12. Santos RE, Grinsztejn B, Peres WAF, Brito PD. Bioimpedância e antropometria na determinação da composição corporal em homens portadores de HIV. Rev bras Nutri Clin. 2016; 31(1): 60-64.

13. Beraldo RA, Santos AP, Guimarães MP, Vassimon HS, Paula FJA, Machado $\mathrm{DRL}$, et al. Redistribuição de gordura corporal e alterações no metabolismo de lipídeos e glicose em pessoas vivendo com HIV/AIDS. Rev bras epidemiol. 2017 Jul; 20(3):526-36. doi: https://doi.org/10.1590/1980-5497201700030014.

14. Montalvo R, Bernabe-Ortiz A, Kirwan DE, Gilman RH. Bioimpedance markers and tuberculosis outcome among hiv-infected patients. Afr J Infect Dis. 2018; 12(2): 47-54. doi: 10.21010/ajid.v12i2.8.

15. Antunes A, Rodrigues A, Geraix J, Silveira L, Pereira P, Carvalhaes M. Nutritional assessment of hospitalized HIV-infected patients by the phase angle z-score measurement. Nutr Hosp. 2012 May-Jun; 27(3): 771-4. doi: 10.3305/ nh.2012.27.3.5684.

16. León AJD, Almeida ARD, Velásquez GS, Salazar GA, Castilho JCM, Castillo DZ. Parámetros de composición corporal en pacientes VIH mediante el análisis de la biompedancia eléctrica. Bol Venez Infectol. 2016 Jan-Jun; 27(1): 21-28.

17. Khalil SF, Mohktar MS, Ibrahim F. Bioimpedance Vector Analysis in Diagnosing Severe and Non-Severe Dengue Patients. Sensors (Basel). 2016 Jun; 16(6): 911. doi: $10.3390 /$ s16060911.

18. Silva TBD e, Libonati RMF. Ângulo de fase e indicadores do estado nutricional em pessoa vivendo com HIV/Aids com síndrome lipodistrófica secundária à terapia antirretroviral/ Phase angle and indicators of nutritional status in a person living with HIV / AIDS with lipodystrophic syndrome secondary to antiretroviral therapy. Bras J Health Review. 2020; 3(4):10710-27. doi: https:// doi.org/10.34119/bjhrv3n4-331.

19. Wady MTB, Linhares-Carvalho MI, Salles-Costa R, Valle J, Castello-Branco LRR. Investigação dos aspectos nutricionais em homens abrigados em uma instituição filantrópica envolvida em surto de tuberculose. Bol. Pneumol. Saint. 2004 Abr; 12(1): 13-8.

20. Werneck GL, Hasselmann MH, Gouvêa TG. Panorama dos estudos sobre nutrição e doenças negligenciadas no Brasil. Ciênc saúde coletiva. 2011 Jan;16(1): 39-62. doi: https://doi.org/10.1590/S1413-81232011000100009.

21. Silva EP da, Nascimento AMV do, Campos JRE, Campos JBR, Barros AB, Luz DCRP. Prevalência de sobrepeso e obesidade em indivíduos com HIV/AIDS: uma revisão integrativa. Nursing. 2021; Abr;24(272):5148-61.

22. Sampaio Ethiane de Jesus. Ângulo de fase em pacientes com HIV/ AIDS: comparação com métodos de avaliação do estado nutricional. 2012. 78 f. Dissertação (Mestrado em Alimentos, Nutrição e Saúde). [Salvador]: Universidade Federal da Bahia; 2012. doi: http://repositorio.ufba.br/ri/handle/ ri/11164.

23. Antunes AA, Rodrigues APAP, Geraix J, Silveira LVA, Pereira PCM, Carvalhaes MABL. Nutritional assessment of hospitalized HIV-infected patients by the phase angle z-score measurement. Nutr Hosp. 2012 May-Jun; 27(3): 771-4. doi: 10.3305/nh.2012.27.3.5684

Como citar este artigo/How to cite this article:

Rocha NO, Araújo AQC, Silva PS, Torres PR, Santos R, Pinto LMC, et al. Bioimpedância em doenças infecciosas: revisão crítica. J Health Biol Sci. 2021; 9(1):1-5. 